\title{
Statement from the North American Society for Cardiovascular Imaging on imaging strategies to reduce the scarcity of healthcare resources during the COVID-19 outbreak
}

\author{
Gregory Kicska ${ }^{1}$ - Diana E. Litmanovich ${ }^{2} \cdot$ Karen G. Ordovas $^{3} \cdot$ Phillip M. Young $^{4} \cdot$ Carole Dennie $^{5} \cdot$ Quynh A. Truong $^{6}$. \\ Suhny Abbara ${ }^{7}$. Jacobo Kirsch ${ }^{8}$ (D)
}

Received: 13 April 2020 / Accepted: 22 April 2020 / Published online: 30 May 2020

C) Springer Nature B.V. 2020

\begin{abstract}
Coronavirus disease 2019 (COVID-19), caused by severe acute respiratory syndrome coronavirus 2 (SARS-CoV-2), is an evolving global pandemic that is predicted to strain healthcare resources at multiple locations throughout North America and the World. As of April 6, 2020, the apex of infection rates is predicted to occur within 1 to 5 weeks at various locations. Widespread reports of personal protective equipment (PPE) shortages, and healthcare worker exposure to disease have become commonplace. To mitigate this crisis, we are suggesting imaging strategies that aim to use the least PPE, require the smallest number of potential staff exposures, and streamlines utilization of imaging. They are broadly organized by (1) substituting a noninvasive diagnostic test in place of a semi-invasive or invasive diagnostic tests, and (2) consolidating diagnostic imaging.
\end{abstract}

Keywords Cardiovascular imaging $\cdot$ COVID19 $\cdot$ Cardiac CT $\cdot$ Cardiac MRI

Gregory Kicska

kicskag@uw.edu

Jacobo Kirsch

kirschj@ccf.org

1 Department of Radiology, University of Washington, Seattle, WA, USA

2 Department of Radiology, Beth Israel Lahey Health, Harvard Medical School, Boston, MA, USA

3 Department of Radiology and Biomedical Imaging, University of California San Francisco, San Francisco, USA

4 Department of Radiology, Mayo Clinic, 200 First Street Southwest, Rochester, MN 55905, USA

5 Department of Medical Imaging, The Ottawa Hospital, University of Ottawa, Ottawa, ON, Canada

6 Department of Radiology and Division of Cardiology, New York-Presbyterian Hospital and Weill Cornell Medicine, New York, NY, USA

7 Department of Radiology, UT Southwestern Medical Center, Dallas, TX, USA

8 Department of Imaging, Cleveland Clinic Florida, Weston, FL, USA

\section{Introduction}

COVID-19 is a global pandemic that is predicted to strain healthcare resources. The apex of disease is predicted to occur between April 15 and May 30 at various locations throughout the United States and Canada. As of April 6, 2020, all U.S. states have yet to reach their apex of infection, but there are already widespread reports of personal protective equipment (PPE) shortages, making it challenging to treat COVID-19 patients. To conserve PPE, hospitals have canceled or postponed elective procedures, which will have unknown downstream effects on population health [1]. PPE shortages also increase the risk that healthcare workers may contract COVID-19, which would force them to self-isolate, further worsening the ability to deliver care [1]. In this crisis, it is important to protect our healthcare workers and their ability to deliver care by considering imaging strategies that use the least PPE, decrease exposure to staff, and streamline utilization of imaging equipment [1-3].

We are suggesting imaging strategies that aim to alleviate the anticipated strain on hospital resources. They are broadly organized by (1) substituting a noninvasive diagnostic test in place of a semi-invasive or invasive diagnostic test, and (2) consolidating multiple diagnostic tests into one single exam. 
Substituting noninvasive diagnostic imaging in place of semi-invasive or invasive diagnostic imaging reduces PPE use and has comparable diagnostic accuracy [4]. In most cases, noninvasive diagnostic imaging is associated with less interactions between the patient and the healthcare worker, which decreases the risk of transmitting a communicable disease such as COVID-19.

Consolidating imaging is another strategy to reduce PPE use and healthcare worker exposure because it may reduce the number of visits a suspected or positive COVID-19 patient makes to the Radiology Department. Consolidation is accomplished by anticipating the future imaging needs of a patient and meeting that need by performing a lower number of more comprehensive studies. This may provide the information likely to be sought later during hospitalization, but with less imaging utilization.

There are varying levels of evidence for our suggested strategies. Suggestions backed by a strong level of evidence may already be routine care at some institutions. For suggestions supported by expert opinion alone, the decision to implement a suggested strategy should depend on the current clinical environment. If hospital resources are not strained, only suggestions with strong evidence may be used. However, if resources are severely strained, suggestions based on expert opinion may be considered. Therefore, we separated suggestions with a strong level of evidence from those based on expert opinion.

This document is organized as follows: (1a) noninvasive diagnostic imaging alternatives supported by strong evidence that can be performed in place of semi-invasive or invasive imaging for specific clinical indications in all patients; (1b) noninvasive diagnostic imaging alternatives supported by expert opinion in place of semi-invasive or invasive imaging in suspected or positive COVID-19 patients; and (2) alternative imaging protocols that can be performed in an attempt to consolidate multiple anticipated imaging exams.

These strategies should only be considered during a time of crisis when healthcare resources are strained. All changes in usual care should consider the additional risks imposed on patients. All changes to usual care should be made after consultation with physicians, administration, and stakeholders, and according to local institutional policies and expertise, balancing the immediate needs of the patient with the obligation to deliver care to the community as a whole.

\section{$\mathrm{NASCI}$ recommendations for the use of noninvasive diagnostic imaging alternatives}

\section{(1a) Noninvasive diagnostic imaging alternatives supported by strong evidence that can be performed in place of semi-invasive or invasive imaging for specific clinical indications in all patients, particularly COVID-19-positive or COVID-19 suspected patients (Table 1)}

Indication 1: Acute chest pain and elevated troponin and equivocal diagnosis of non-ST elevation myocardial infarction (NSTEMI).

Rationale: This approach decreases utilization of diagnostic invasive coronary angiography in the catheterization laboratory, which requires airborne precautions, and replaces the assessment with coronary Computed Tomography (CT), a droplet precaution test [5]. In COVID-19-positive or COVID-19 suspected patients, coronary CT can be used to rule out coronary artery disease as the cause of acute chest pain leaving myocarditis, possibly due to COVID-19, as the leading diagnosis. If coronary artery disease is ruled out, this approach changes management by replacing intensive acute coronary syndrome care with supportive care for acute myocarditis.

Evidence: In patients with suspected acute coronary syndrome (acute chest pain and/or elevated troponin) and equivocal NSTEMI diagnosis, a negative coronary CT may be used to exclude the diagnosis of acute coronary syndrome

Table 1 Alternative imaging in specific clinical scenarios

\begin{tabular}{lll}
\hline Alternative imaging in specific clinical scenarios & & \\
\hline Indication & Usual care & Suggested protocol \\
\hline Elevated troponin and equivocal diagnosis of NSTEMI & Invasive coronary angiography & Coronary CT \\
Acute chest pain, negative initial troponin, intermediate risk & Invasive coronary angiography, or & Coronary CT \\
& $24 \mathrm{~h}$ serial troponin + EKG & Cardiac CT with delayed phase \\
Exclusion of LAA thrombus prior to urgent cardioversion & TEE & CTA \\
Emergent TAVR or SAVR planning & TEE & Cardiac CT \\
Prosthetic or native heart valve dysfunction or suspected endocarditis & TEE & \\
\hline
\end{tabular}

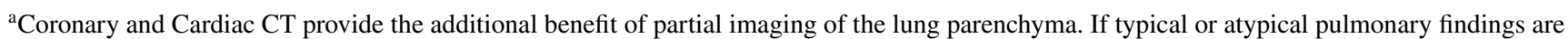
encountered, consultation with a radiologist with thoracic expertise is encouraged, and appropriate documentation and timely communication of these findings is essential, especially in cases not known or suspected to have the disease [18] 
(ACS), and favor the possibility of non-coronary etiologies, primarily myocarditis $[6,7]$.

Indication 2: Acute chest pain in patients with negative initial troponin, and intermediate risk for coronary artery disease.

Rationale: This approach accelerates patient discharge by replacing the standard of care which entails $24 \mathrm{~h}$ observation, serial enzymes, and EKG, and usually performed in an observation unit, Emergency Department (ED) or inpatient setting. Decreased length of hospital stay limits exposure of healthcare workers and frees beds for COVID-19 patients in need of in-hospital care. Coronary CT can be used to rule out coronary artery disease as the cause of acute chest pain, leaving myocarditis, possibly due to COVID-19, as the leading diagnosis.

Evidence: Randomized controlled trials $[8,9]$ and observational studies [10] have shown that a coronary CT-guided approach has similar safety outcomes and decreased length of hospital stay compared to standard of care in intermediate risk patients with negative initial troponin.

Indication 3: Patients in need of urgent cardioversion with indication to rule out left atrial appendage (LAA) thrombus for stroke prevention.

Rationale: This approach replaces transesophageal echocardiography (TEE), which requires airborne precautions, with cardiac CT with delayed phase (droplet precautions) to exclude the presence of an LAA thrombus in patients with arrhythmias. Cardiac CT with delayed phase can be used to rule out the presence of LAA thrombus.

Evidence: Cardiac CT with delayed images has comparable sensitivity and specificity to TEE to exclude the presence of LAA thrombus [11]. Studies utilizing cardiac CT instead of TEE in the imaging workup for LAA thrombus before left atrial (LA) ablation have shown no difference in adverse outcomes such as stroke or embolic events $[12,13]$.

Indication 4: Patients with severe aortic stenosis with cardiac decompensation, in need of acute aortic valve replacement, surgical, or transcatheter.

Rationale: CT angiography (CTA) in patients in need of urgent Transcatheter Aortic Valve Replacement (TAVR) or Surgical Aortic Valve Replacement (SAVR) can replace TEE for assessment of TAVR eligibility. CTA approach would reduce the exposure from airborne to droplet precautions. Dedicated pre-TAVR CTA could guide the selection of the type of intervention and might preclude the need for preprocedure TEE [14]. This imaging also includes the added benefit of evaluating proximal coronary arteries for stenosis $[15,16]$.

Evidence: CTA is an accurate imaging modality to determine eligibility for TAVR based on valve sizing and peripheral arterial access with evaluation of coronary arteries [17].

Indication 5: Patients with acute symptomatic prosthetic or native heart valve dysfunction or suspected endocarditis.
Rationale: Cardiac CT with retrospective gating can delineate prosthetic heart valve morphology and identify the reason for cardiac decompensation, such as the presence of a vegetation or thrombus. Cardiac CT can determine if the perivalvular region is involved and thus influence a change in management from medical therapy to surgical intervention. Urgent Cardiac CT with functional analysis may provide sufficient information to guide the next treatment step without the need for TEE.

Evidence: Cardiac CT functional native or prosthetic valve assessment has similar accuracy to TEE for diagnosis of endocarditis or vegetations [18].

\section{(1b) Noninvasive diagnostic imaging alternatives supported by expert opinion in place of semi-invasive or invasive imaging for indications other than listed in 1a and only in COVID-19-positive or COVID-19 suspected patients (Table 2 )}

There are multiple clinical scenarios that require imaging with diagnostic invasive coronary angiography or TEE in a patient with COVID-19 or suspected COVID-19 that are not listed in Sect. 1a. On a case-by-case basis, it may be determined as reasonable to perform Coronary or Cardiac CT in place of these other semi-invasive or invasive tests. Because this substitution is considered reasonable, but evidence related to specific clinical indications is not present, these recommendations are based on expert opinion. Therefore, a decision to employ these protocols should be based on clinical need in the setting of the evolving crisis. For example, the protocols may be used differently in COVID-19 patients who are severely ill versus stable.

1. Substitution of coronary CT for catheter coronary angiography in known COVID-19 and COVID-19-suspected patients.

Rationale: This strategy leads to conservation of PPE. It reduces risk of transmitting infection to healthcare workers

Table 2 General recommendations for noninvasive alternatives to semi-invasive or invasive imaging

Noninvasive alternatives to semi-invasive or invasive imaging ${ }^{\mathrm{a}}$

\begin{tabular}{ll}
\hline Usual care & Suggested protocol \\
\hline Diagnostic invasive coronary angiography & Coronary CT \\
TEE & Cardiac CT, with or \\
& without delayed \\
& phase \\
\hline
\end{tabular}

${ }^{a}$ Coronary and Cardiac CT provide the additional benefit of partial imaging of the lung parenchyma. If typical or atypical pulmonary findings are encountered, consultation with a radiologist with thoracic expertise is encouraged, and appropriate documentation and timely communication of these findings is essential, especially in cases not known or suspected to have the disease [18] 
and reduces time for disinfecting the imaging suite compared to angiography suite, which maximizes availability of diagnostic services. Cleaning after a CT examination is based on droplet precautions, whereas diagnostic angiography and TEE are based on airborne precautions, a more time intensive cleaning process.

2. Substitution of cardiac CT for TEE in known COVID19 and COVID-19-suspected patients.

Rationale: Same as above.

\section{(2) Alternative imaging that can be performed in an attempt to consolidate imaging, supported by expert opinion, in COVID-19-positive or COVID-19 suspected patients (Table 3 )}

If possible, the number of CT imaging exams should be consolidated (reduced) in known COVID-19 and COVID19-suspected patients by changing the imaging protocol to a more comprehensive.

Rationale: Hospitalized patients often have multiple CT examinations performed during their hospitalization, sometimes in rapid succession. Each of these examinations requires resources and exposes clinical staff to the risk of infection. If a crisis situation is present, and resources are strained, it may be advantageous to predict future imaging needs and attempt to consolidate imaging by ordering a more comprehensive exam in place of multiple exams. For example, a Triple Rule-Out (TRO) protocol CT likely yields all the information contained in a CT Pulmonary Angiogram (CTPA), with the added evaluation of the aorta and coronary arteries. Likewise, a TRO protocol CT is likely to evaluate coronary arteries as well as a coronary CT, with the added benefit of evaluating for aortic disease, pulmonary embolism, and the entire lung for pulmonary parenchymal disease.

Table 3 List of protocols that can be used for consolidating imaging Consolidation of imaging studies

\begin{tabular}{lc}
\hline Original & Consolidated \\
\hline $\begin{array}{l}\text { Coronary CT, Cardiac CT, CTA, CTPA, CT Chest } \\
\text { Unenhanced }\end{array}$ & TRO \\
CT Chest unenhanced & CT Chest with \\
& contrast, \\
& TRO, CTA, \\
& or CTPA \\
CT Chest with contrast & Cannot be \\
& consolidated \\
& with another \\
& exam \\
&
\end{tabular}

The information obtained by imaging with a protocol listed in the "original" column is likely to be provided by a protocol listed under the "consolidated" column
Extending the information routinely gathered from a coronary CT, CTA or CTPA is pertinent in COVID-19 patients because studies have suggested a high incidence of myocarditis in COVID-19 and other studies have reported an increased incidence of pulmonary embolism in viral pneumonia [24].

As an example, myocarditis has been described as common in COVID-19 patients with ARDS or who are severely ill and is associated with a poor prognosis $[19,20]$. The diagnosis can be suggested by an elevated troponin in a patient with no known history of cardiac disease. However, in some cases, myocardial ischemia from epicardial coronary disease may still need to be excluded. In that setting, a Coronary CT may be used to evaluate the coronary arteries. However, if the lung parenchyma also needs to be evaluated, a TRO study may provide that needed additional information.

Substituting one study for another should be decided on a case-by-case basis, and considered only if appropriate resources and expertise are available. The risks associated with the protocol change should also be considered. Adding intravenous contrast to an examination is associated with risk, and an individual patient's renal status and potential for allergic reaction should be considered. Anecdotally, COVID-19 patients may suffer increased rates of renal dysfunction, and this should be considered before administering contrast. Using cardiac gating may increase radiation and duration in the radiology suite interacting with technologists. Nitroglycerin and beta-blockers are not required for TRO imaging but may be used if it is justified by the risk/benefit ratio of a given patient. In summary, cardiac-gating and cardiac medications should only be employed if it is likely to obviate the need for a future study and is an acceptable risk/ benefit for the patient.

\section{Additional information}

\section{Summary of other Society guidelines or statements}

Several societies have contributed guidelines or strategies related to imaging patients with COVID-19. Most of these recommendations have focused on changing the first-line imaging study for purposes of protecting staff, cleaning equipment, and deferment of non-emergent imaging studies to a later date. These guidelines have not addressed imaging for the purpose of reducing scarcity of resources.

A brief summary of society statements is included below.

Radiological Society of North America, American College of Radiology, Society of Thoracic Radiology [21]

- Guidelines for reporting CT findings in COVID-19 patients 
- Radiology Department Preparedness for COVID-19: Radiology Scientific Expert Panel

\section{Society of Cardiac Computed Tomography [22]}

- PPE use and equipment cleaning when patients with confirmed or suspected COVID-19 are imaged.

- Guidelines for delaying imaging for specific cardiac indications

- Substitution of noninvasive diagnostic imaging for other forms of imaging to reduce exposure

\section{American Society of Echocardiography [23]}

- PPE used for echocardiography

- Guidelines for delaying imaging for specific cardiac indications

- Substitution of noninvasive diagnostic imaging for other forms of imaging to reduce exposure

American College of Cardiology's Interventional Council and the Society of Cardiovascular Angiography and Intervention [5]

- Recommendation to deferred elective cardiac catheterization cases for the purposes of conserving PPE

\section{Group definitions}

COVID-19 patients

Suspected COVID-19 patients

Non-COVID-19 patients
Patients with a positive RT-PCR result within the last 14 days or patients within RT-PCR result over 14 days ago, but still experiencing symptoms believed to be caused by COVID-19

Patients with unexplained lower respiratory tract symptoms and a pending or single negative RT-PCR.

Patients without lower respiratory tract symptoms or with lower respiratory tract symptoms but a negative RT-PCR result for COVID-19 and an alternative source of infection.

\section{Protocol definitions}

Diagnostic angiography

Coronary $C T$

Cardiac CT:

CT angiography (CTA)

CT chest unenhanced

CT chest with contrast

CT pulmonary angio graphy (CTPA)

Triple-rule-out (TRO)

\section{Compliance with ethical standards}

Conflict of interest Authors report no conflict of interest.
Invasive catheter-based angiography for imaging only and not intervention.

ECG-gated CT of the heart and proximal aorta with contrast injection optimized for coronary artery of enhancement. Consider prefacing this study with ECG-gated Coronary calcium score CT.

ECG-gated Cardiac CT with contrast injection optimized for imaging of cardiac chambers and/or valve morphology with or without 90 s delay. Thoracic CT with contrast injection optimized for systemic arterial enhancement (i.e. aortic).

Thoracic CT without intraveThoracic CT with intravenous contrast administration, usually imaging at a $70 \mathrm{~s}$ delay.

Thoracic CT with intravenous contrast administration optimized for pulmonary artery enhancement. ECG-gated thoracic CT with intravenous contrast administration optimized for imaging of pulmonary and coronary arteries, and aorta. nous contrast administration.

\section{References}

1. Resources for clinics and healthcare facilities I CDC. Resources for clinics and healthcare facilities I CDC. https://www.cdc. gov/coronavirus/2019-ncov/healthcare-facilities/index.html. Accessed 28 March 2020 
2. Kooraki S, Hosseiny M, Myers L, Gholamrezanezhad A (2020) Coronavirus (COVID-19) outbreak: what the Department of Radiology Should Know. J Am Coll Radiol. United States

3. COVID-19: strategies for optimizing the supply of PPE I CDC. COVID-19: strategies for optimizing the supply of PPE I CDC. https://www.cdc.gov/coronavirus/2019-ncov/hcp/ppe-strategy/ index.html. Accessed 28 March 2020

4. Transmission-based precautions | Basics | Infection Control | CDC". Transmission-based precautions I Basics I Infection Control I CDC". https://www.cdc.gov/infectioncontrol/basics/trans mission-based-precautions.html. Accessed 28 March 2020

5. Welt FGP, Shah PB, Aronow HD, Bortnick AE, Henry TD, Sherwood MW, Young MN, Davidson LJ, Kadavath S, Mahmud E, Kirtane AJ, American College of Cardiology's (ACC) Interventional Council and the Society of Cardiovascular Angiography and Intervention (SCAI) (2020) Catheterization Laboratory Considerations During the Coronavirus (COVID-19) Pandemic: From ACC's Interventional Council and SCAI. J Am Coll Cardiol. United States

6. Guichard JL, Hage FG (2016) Guidelines in review: 2015 ACR/ ACC/AHA/AATS/ACEP/ASNC/NASCI/SAEM/SCCT/SCMR/ SCPC/SNMMI/STR/STS Appropriate Utilization of Cardiovascular Imaging in Emergency Department Patients with Chest Pain: a joint document of the American College of Radiology Appropriateness Criteria Committee and the American College of Cardiology Appropriate Use Criteria Task Force. J Nucl Cardiol 23(5):1142-1146

7. Linde JJ, Kelbæk H, Hansen TF, Sigvardsen PE, Torp-Pedersen C, Bech J, Heitmann M, Nielsen OW, Høfsten D, Kühl JT, Raymond IE, Kristiansen OP, Svendsen IH, Vall-Lamora MHD, Kragelund C, de Knegt M, Hove JD, Jørgensen T, Fornitz GG, Steffensen R, Jurlander B, Abdulla J, Lyngbæk S, Elming H, Therkelsen SK, Jørgensen E, Kløvgaard L, Bang LE, Hansen PR, Helqvist S, Galatius S, Pedersen F, Abildgaard U, Clemmensen P, Saunamäki K, Holmvang L, Engstrøm T, Gislason G, Køber LV, Kofoed KF (2020) Coronary CT angiography in patients with non-ST-segment elevation acute Coronary Syndrome. J Am Coll Cardiol 75(5):453-463

8. Litt HI, Gatsonis C, Snyder B, Singh H, Miller CD, Entrikin DW, Leaming JM, Gavin LJ, Pacella CB, Hollander JE (2012) CT angiography for safe discharge of patients with possible acute coronary syndromes. N Engl J Med 366(15):1393-1403

9. Hoffmann U, Truong QA, Schoenfeld DA, Chou ET, Woodard PK, Nagurney JT, Pope JH, Hauser TH, White CS, Weiner SG, Kalanjian S, Mullins ME, Mikati I, Peacock WF, Zakroysky P, Hayden D, Goehler A, Lee H, Gazelle GS, Wiviott SD, Fleg JL, Udelson JE, ROMICAT-II Investigators (2012) Coronary CT angiography versus standard evaluation in acute chest pain. N Engl J Med 367(4):299-308

10. Takakuwa KM, Keith SW, Estepa AT, Shofer FS (2011) A metaanalysis of 64-section coronary CT angiography findings for predicting 30-day major adverse cardiac events in patients presenting with symptoms suggestive of acute coronary syndrome. Acad Radiol 18(12):1522-1528

11. Romero J, Husain SA, Kelesidis I, Sanz J, Medina HM, Garcia MJ (2013) Detection of left atrial appendage thrombus by cardiac computed tomography in patients with atrial fibrillation: a meta-analysis. Circ Cardiovasc Imaging. 6(2):185-194

12. Bilchick KC, Mealor A, Gonzalez J, Norton P, Zhuo D, Mason P, Ferguson JD, Malhotra R, Michael Mangrum J, Darby AE, DiMarco J, Hagspiel K, Dent J, Kramer CM, Stukenborg GJ, Salerno M (2016) Effectiveness of integrating delayed computed tomography angiography imaging for left atrial appendage thrombus exclusion into the care of patients undergoing ablation of atrial fibrillation. Heart Rhythm 13(1):12-19
13. Mosleh W, Sheikh A, Said Z, Ahmed MA, Gadde S, Shah T, Wilson MF, Beck H, Kim C, Sharma UC (2018) The use of cardiac-CT alone to exclude left atrial thrombus before atrial fibrillation ablation: efficiency, safety, and cost analysis. Pacing Clin Electrophysiol 41(7):727-733

14. Andreini D, Pontone G, Mushtaq S, Bartorelli AL, Ballerini G, Bertella E, Segurini C, Conte E, Annoni A, Baggiano A, Formenti A, Fusini L, Tamborini G, Alamanni F, Fiorentini C, Pepi M (2014) Diagnostic accuracy of multidetector computed tomography coronary angiography in 325 consecutive patients referred for transcatheter aortic valve replacement. Am Heart J. 168(3):332-339

15. Hamdan A, Wellnhofer E, Konen E, Kelle S, Goitein O, Andrada B, Raanani E, Segev A, Barbash I, Klempfner R, Goldenberg I, Guetta V (2015) Coronary CT angiography for the detection of coronary artery stenosis in patients referred for transcatheter aortic valve replacement. J Cardiovasc Comput Tomogr 9(1):31-41

16. Annoni AD, Andreini D, Pontone G, Mancini ME, Formenti A, Mushtaq S, Baggiano A, Conte E, Guglielmo M, Muscogiuri G, Muratori M, Fusini L, Trabattoni D, Teruzzi G, Coutinho Santos AI, Agrifoglio M, Pepi M (2018) CT angiography prior to TAVI procedure using third-generation scanner with wide volume coverage: feasibility, renal safety and diagnostic accuracy for coronary tree. Br J Radiol 91(1090):20180196

17. Blanke P, Weir-McCall JR, Achenbach S, Delgado V, Hausleiter J, Jilaihawi H, Marwan M, Nørgaard BL, Piazza N, Schoenhagen P, Leipsic JA (2019) Computed tomography imaging in the context of transcatheter aortic valve implantation (TAVI)/ transcatheter aortic valve replacement (TAVR): an expert consensus document of the Society of Cardiovascular Computed Tomography. JACC Cardiovasc Imaging 12(1):1-24

18. Doherty JU, Kort S, Mehran R, Schoenhagen P, Soman P, Appropriate Use Criteria Task Force. ACC/AATS/AHA/ASE/ ASNC/HRS/SCAI/SCCT/SCMR/STS (2019) Appropriate use criteria for multimodality imaging in the assessment of cardiac structure and function in Nonvalvular Heart Disease : a report of the American College of Cardiology appropriate use criteria task force, American Association for Thoracic Surgery, American Heart Association, American Society of Echocardiography, American Society of Nuclear Cardiology, Heart Rhythm Society, Society for Cardiovascular Angiography and Interventions, Society of Cardiovascular Computed Tomography, Society for Cardiovascular Magnetic Resonance, and the Society of Thoracic Surgeon. J Nucl Cardiol. 2019:1392-1413

19. Guo T, Fan Y, Chen M, Wu X, Zhang L, He T, Wang H, Wan J, Wang X, Lu Z (2020) Cardiovascular implications of fatal outcomes of patients with Coronavirus Disease 2019 (COVID19). JAMA Cardiol

20. Li B, Yang J, Zhao F, Zhi L, Wang X, Liu L, Bi Z, Zhao Y (2020) Prevalence and impact of cardiovascular metabolic diseases on COVID-19 in China. Clin Res Cardiol

21. Simpson S, Kay FU, Abbara S, Bhalla S, Chung JH, Chung M, Henry TS, Kanne JP, Kligerman S, Ko JP, Litt H (2020) Radiological Society of North America Expert Consensus Statement on Reporting Chest CT Findings Related to COVID-19. Endorsed by the Society of Thoracic Radiology, the American College of Radiology, and RSNA. Radiology 2:2. https://doi. org/10.1148/ryct.2020200152

22. Choi AD, Abbara S, Branch KR, Feuchtner GM, Ghoshhajra B, Nieman K, Pontone G, Villines TC, Williams MC, Blankstein R (2020) Society of cardiovascular computed tomography guidance for use of cardiac computed tomography amidst the COVID-19 pandemic. J Cardiovasc Comput TomogrP 
23. ASE Statement on COVID-19. ASE Statement on COVID-19. https://www.asecho.org/ase-statement-covid-19/. Accessed 28 March 2020

24. Bunce PE, High SM, Nadjafi M, Stanley K, Liles WC, Christian MD (2011) Pandemic H1N1 influenza infection and vascular thrombosis. Clin Infect Dis 52(2):e14-e17
Publisher's Note Springer Nature remains neutral with regard to jurisdictional claims in published maps and institutional affiliations. 